\title{
Siglec-7 is a predictive biomarker for the efficacy of cancer vaccination against metastatic colorectal cancer
}

\author{
KENSUKE YAMADA $^{1}$, SHOICHI HAZAMA ${ }^{1,2}$, NOBUAKI SUZUKI ${ }^{1}$, MING XU $^{1}$, YUKI NAKAGAMI ${ }^{1,2}$, \\ NOBUYUKI FUJIWARA ${ }^{1}$, RYOUICHI TSUNEDOMI ${ }^{1}$, SHIN YOSHIDA ${ }^{1}$, SHINOBU TOMOCHIKA ${ }^{1}$, \\ SATOSHI MATSUKUMA ${ }^{1}$, HIROTO MATSUI ${ }^{1}$, YUKIO TOKUMITSU ${ }^{1}$, SHINSUKE KANEKIYO ${ }^{1}$, \\ YOSHITARO SHINDO $^{1}$, YUSAKU WATANABE ${ }^{1}$, MICHIHISA IIDA ${ }^{1}$, SHIGERU TAKEDA ${ }^{1}$, \\ TATSUYA IOKA ${ }^{3}$, TOMIO UENO ${ }^{4}$, HIROYUKI OGIHARA ${ }^{5}$, YOSHIHIKO HAMAMOTO ${ }^{5}$, YOSHINOBU HOSHII ${ }^{6}$, \\ HIROO KAWANO $^{7}$, TOMONOBU FUJITA ${ }^{8}$, YUTAKA KAWAKAMI ${ }^{8}$ and HIROAKI NAGANO ${ }^{1}$ \\ ${ }^{1}$ Department of Gastroenterological, Breast and Endocrine Surgery, Yamaguchi University Graduate School of Medicine; \\ ${ }^{2}$ Department of Translational Research and Developmental Therapeutics Against Cancer, \\ Yamaguchi University School of Medicine; ${ }^{3}$ Oncology Center, Yamaguchi University Hospital, Ube, Yamaguchi 755-8505; \\ ${ }^{4}$ Department of Gastroenterological Surgery, Kawasaki Medical School, Kurashiki, Okayama 701-0192; \\ ${ }^{5}$ Division of Electrical, Electronic and Information Engineering, Graduate School of Sciences and Technology for Innovation, \\ Yamaguchi University, Ube, Yamaguchi 755-8611; ${ }^{6}$ Department of Diagnostic Pathology, Yamaguchi University Hospital; \\ ${ }^{7}$ Department of Basic Laboratory Sciences, Yamaguchi University Graduate School of Medicine, Ube, Yamaguchi 755-8505; \\ ${ }^{8}$ Division of Cellular Signaling, Institute for Advanced Medical Research, \\ Keio University School of Medicine, Tokyo 160-8582, Japan
}

Received May 28, 2020; Accepted September 29, 2020

DOI: $10.3892 / 01.2020 .12271$

\begin{abstract}
Cancer immunotherapy, including vaccination, is considered a major scientific and medical breakthrough. However, cancer immunotherapy does not result in durable objective responses against colorectal cancer (CRC). To improve the efficacy of immunotherapy, the present study investigated several biomarkers for selecting patients who were expected to respond well to immunotherapy. Firstly, a comprehensive proteomic analysis was performed using tumor tissue lysates from patients enrolled in a phase II study, in which five human leukocyte antigen (HLA)-A*24:02-restricted peptides were administered. Sialic acid-binding immunoglobulin type lectin (Siglec)-7 was identified as a potential predictive biomarker. Subsequently, this biomarker was validated using western blot analysis, and immunofluorescence using tissue samples from the patients enrolled in the phase II study. The expression levels of Siglec-7 detected by immunofluorescence were quantified and their association with overall survival (OS) in patients treated with the peptide vaccine was
\end{abstract}

Correspondence to: Professor Hiroaki Nagano, Department of Gastroenterological, Breast and Endocrine Surgery, Yamaguchi University Graduate School of Medicine, 1-1-1 Minami-Kogushi, Ube, Yamaguchi 755-8505, Japan

E-mail: hnagano@yamaguchi-u.ac.jp

Key words: colorectal cancer, sialic acid-binding immunoglobulin type lectin-7, biomarker, peptide vaccine, protein expression analysis examined. Furthermore, considering the important role of tumor-infiltrating lymphocytes (TILs) for CRC prognosis, the densities of $\mathrm{CD}^{+}, \mathrm{CD}^{+}{ }^{+} \mathrm{CD}^{+}$and forkhead box P3 (FOXP3) ${ }^{+}$ $\mathrm{T}$ cells in CRC tissues were examined and compared with Siglec-7 expression. The mean expression levels of Siglec-7 were significantly higher in patients with poor prognosis, with an OS of $\leq 2$ years, as shown in comprehensive proteomic analysis $(\mathrm{P}=0.016)$ and western blot analysis $(\mathrm{P}=0.025)$. Immunofluorescence analysis demonstrated that Siglec-7 was expressed in intratumoral macrophages. The OS in patients with high Siglec-7 expression was significantly shorter than in that in patients with low Siglec-7 expression $(\mathrm{P}=0.017)$ in the HLA-A*24:02-matched patients. However, this difference was not observed in the HLA-unmatched patients. There was no significant difference in OS between patients according to the numbers of TILs, nor significant correlation between TILs and Siglec-7 expression. In conclusion, Siglec-7 expression in macrophages in tumor tissue may be a novel predictive biomarker for the efficacy of immunotherapy against metastatic CRC.

\section{Introduction}

Colorectal cancer (CRC) is the third most common cause of cancer-related mortality among both men and women (1). In the past decade, chemotherapy and molecular targeted treatment have improved the overall survival (OS) in patients with metastatic CRC to 30 months (2). These drugs, however, have some limitations, including drug resistance and side effects, so the development of new therapeutic options to prevent 
metastatic spread and eventually improve patient survival is necessary (3).

Cancer immunotherapy is considered as a major scientific and medical breakthrough (4), and several immune checkpoint-directed antibodies have increased the OS in patients with various cancers and are approved by the Food and Drug Administration (5,6). For example, PD-1 inhibitor nivolumab and pembrolizumab are used for deficient mismatch repair (dMMR) or microsatellite-instability-high (MSI-H) CRC world-wide $(7,8)$.

However, immunotherapies, including immune checkpoint inhibitors to proficient-MMR CRC, have not achieved durable objective responses against CRC $(9,10)$. Improving the efficacy of immunotherapies requires two approaches. One is the use of combination therapy to alter 'cold tumors' which are characterized by the absence of $\mathrm{T}$ cell infiltration, to 'hot tumors' characterized by the accumulation of proinflammatory cytokines and $\mathrm{T}$ cell infiltration $(5,11)$. The other is the identification of biomarkers to select patients who are expected to respond well to immunotherapy.

The authors of the present study previously reported phase I and II studies in which five epitope peptides were administered to advanced-stage CRC patients $(12,13)$. In these studies, a low neutrophil/lymphocyte ratio and a low plasma interleukin (IL)-6 level were the potential markers for improved survival time of vaccinated patients $(14,15)$. Furthermore, it was also shown that several miRNAs and the integrity of plasma cell-free DNA were predictive biomarkers for active immunotherapy using epitope peptides (15-18).

This study aimed to identify novel predictive biomarkers to select patients who are highly responsive to immunotherapy to improve the efficacy of immunotherapy. To this end, a comprehensive analysis of proteins in tumor tissues was performed and sialic acid-binding immunoglobulin type lectin (Siglec)-7 was identified as a potential predictive biomarker for immunotherapy.

Siglecs are a family of transmembrane receptors predominantly found in both innate and adaptive immune cells, involved in distinguishing between self and non-self-cells by recognizing sialic acids at the cellular surface $(19,20)$. Siglec-7, the seventh member of the Siglec family, is mainly expressed on natural killer (NK) cells, monocytes, macrophages, and a minor subset of $\mathrm{CD}^{+} \mathrm{T}$ cells $(21,22)$, and acts as an inhibitory receptor. The cytoplasmic portion of Siglec-7 contains immune receptor tyrosine-based inhibition motifs (ITIMs), which provide inhibitory signals by recruiting the SH2-domain-containing tyrosine phosphatase (SHP)-1 and SHP2 (22). SHP1 and SHP2 inhibit NK cell activation pathways such as the NKG2D pathway, suppressing NK cell cytotoxicity to tumor cells (23). However, it has never been evaluated for its possible role in cancer immunotherapy. In the present study, Siglec-7 was evaluated for its potential role as a novel biomarker for active immunotherapy.

\section{Materials and methods}

Summary of the phase II study. To assess the clinical benefits of cancer vaccination treatment, a phase II study was conducted using five human leukocyte antigen (HLA )-A*24:02-restricted peptides, including kinase of the outer chloroplast membrane 1 (KOC1) (24), translocase of outer mitochondrial membrane 34 (TOMM34) (25), ring finger protein 43 (RNF43) (26), vascular endothelial growth factor receptor (VEGFR) 1 and 2 (27,28). This phase II study was a non-randomized, HLA-A status double-blind study. The detailed protocol of this phase II study was previously described (13). Briefly, the therapy consisted of a cocktail of five therapeutic epitope peptides in addition to oxaliplatin-containing chemotherapy. The cocktail containing $3 \mathrm{mg}$ of each of the five peptides was mixed with $1.5 \mathrm{ml}$ of incomplete Freund's adjuvant and administered subcutaneously into the thigh or axilla regions every week for 13 weeks, followed by the vaccination once every 2 weeks. Patients $\geq 20$ years old with histologically confirmed advanced CRC who were chemotherapy-naïve, who had adequate functions of critical organs, and had a life expectancy of $\geq 3$ months were eligible. Between February 2009 and November 2012, 96 chemotherapy-naïve CRC patients were enrolled with masked HLA-A*24:02 status.

Sample collection. From the 96 patients who were enrolled in the phase II trial (50 were HLA-A*24:02-matched and 46 were unmatched), 63 formalin-fixed paraffin-embedded (FFPE) tissue samples of primary CRC were obtained (32 were HLA-A*24:02-matched and 31 were unmatched) (Fig. 1). In 14 of the 32 HLA-A*24:02-matched patients, fresh tissues were also snap-frozen in liquid nitrogen and preserved at $-80^{\circ} \mathrm{C}$ until further examination. Primary CRC tissues were obtained by surgery prior to the vaccine treatment at Yamaguchi University Hospital and affiliated hospitals. All samples were obtained with the patients' written informed consent. This study was conducted according to the Declaration of Helsinki and was approved by the Institutional Ethics Review Boards of Yamaguchi University (approval no. H20-102; Clinical Trials Registry: UMIN000001791).

Comprehensive proteomic analysis of tumor tissue. A comprehensive analysis of the protein levels in tumor tissue lysate was performed using the SOMAscan (SomaLogic, Inc.) to quantify 1,129 biologically relevant proteins as previously described (29). Frozen CRC tissue samples were available from patients who survived for either more than three years or less than two years (Fig. 1). According to the manufacturer's protocol (SomaLogic, Inc.), the total protein of the frozen CRC tissue sample was extracted with lysis buffer T-PER Tissue Protein Extraction Reagent (Thermo Fisher Scientific, Inc.) supplemented with Halt Protease Inhibitor Cocktail (Thermo Fisher Scientific, Inc.) through a Qiagen TissueLyser (Qiagen). Samples were sent to SomaLogic and analyzed using the SOMAscan assay. In this assay, protein signals were converted to nucleotide signals using chemically modified nucleotides so that quantification could be done using relative fluorescence signal on microarrays. For this reason, SOMAscan measurements were presented as relative fluorescence units (RFUs).

Western blot analysis. Western blot analysis was performed as previously described (30), using the same extracts as those used in the comprehensive analysis of SOMAscan. Briefly, protein samples $(10 \mu \mathrm{g})$ were separated on $10 \%$ SDS-PAGE and transferred onto a PVDF membrane (Bio-Rad Laboratories, Inc.). 


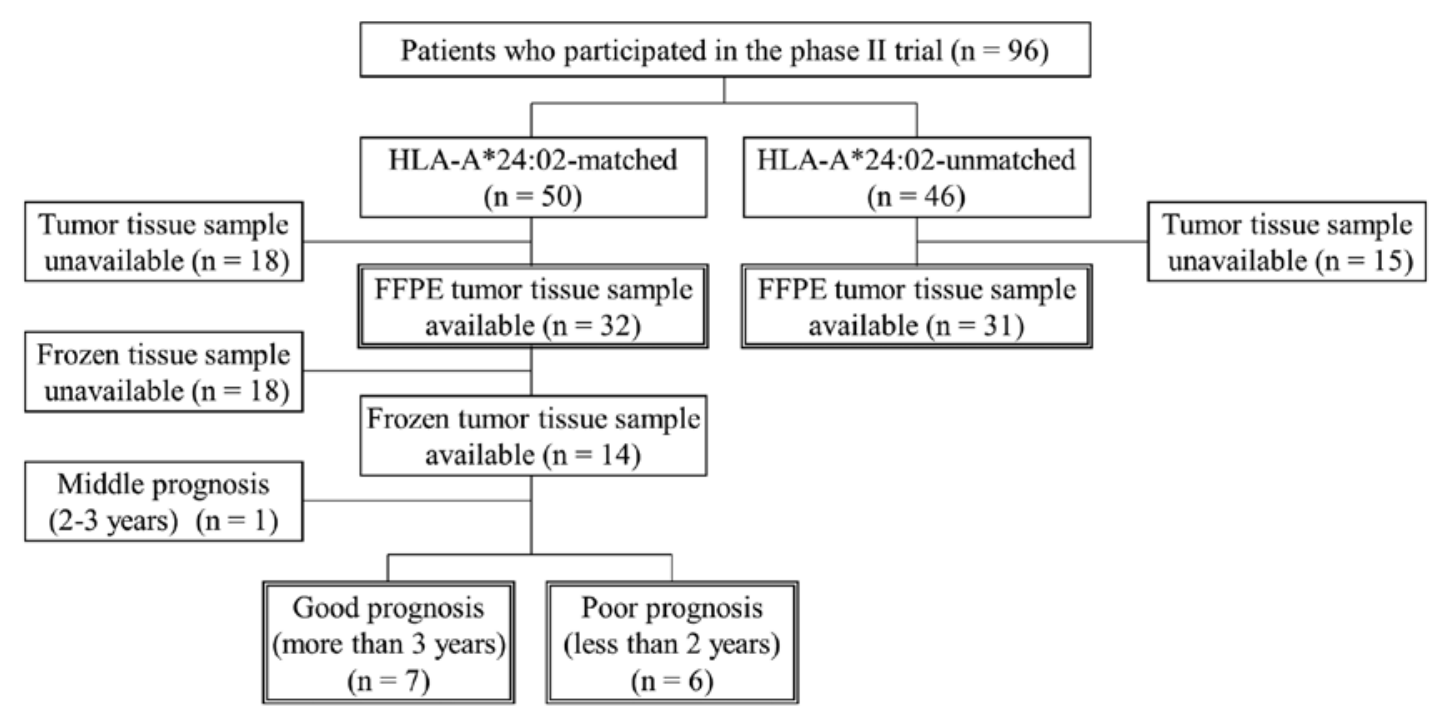

Figure 1. CONSORT diagram of the analyses in this study. Thirteen frozen tissue samples from HLA-A*24:02-matched patients were analyzed using SOMAscan, the comprehensive proteomic analysis; and 63 FFPE tumor tissue samples, 32 HLA-matched and 31 HLA-unmatched patients, were analyzed using immunofluorescence. HLA, human leukocyte antigen; FFPE, formalin-fixed paraffin-embedded.

Membranes were blocked by pre-incubation with 3\% skim milk for $30 \mathrm{~min}$ at room temperature and then were incubated with anti-Siglec-7 antibody (ProteinTech Group, Inc.) at $4^{\circ} \mathrm{C}$ overnight. After washing 3 times with Tris-buffered saline with Tween-20 (TBST) buffer, the membranes were incubated with the corresponding secondary antibody for $1 \mathrm{~h}$ at room temperature. Immunoreactions were detected using an enhanced chemiluminescence (ECL) western blotting detection system and an Amersham Imager 600 (GE Healthcare Life Sciences). Densitometry analysis was performed using ImageJ software (National Institutes of Health) (31). Since the protein levels of VCP, one of the housekeeping proteins, are more stable compared to other housekeeping proteins, such as glyceraldehyde 3-phosphate dehydrogenase (GAPDH) and actin, VCP was chosen as the loading control $(30,32,33)$.

Immunohistochemistry. Immunohistochemistry was carried out on 4- $\mu \mathrm{m}$-thick FFPE sections. For staining Siglec-7, sections were deparaffinized through xylene and graded alcohols, and antigen retrieval was performed in $10 \mathrm{mM}$ Tris-EDTA buffer pH 9.0 (Dako) in a microwave at $95^{\circ} \mathrm{C}$ for $20 \mathrm{~min}$. Endogenous peroxidase activity in the sections was blocked with $3 \%$ hydrogen peroxidase for $20 \mathrm{~min}$, and nonspecific protein binding was blocked with Protein Block Serum-Free (Dako) for $10 \mathrm{~min}$. The staining procedures were performed in a Dako Autostainer (Dako) according to the manufacturer's protocol. Sections were incubated with an anti-Siglec-7 antibody (rabbit polyclonal, 13939-1-AP, ProteinTech Group, Inc.; dilution 1:800) at room temperature for $1 \mathrm{~h}$. After washing 3 times with phosphate-buffered saline (PBS), the sections were incubated with the corresponding secondary antibody for $30 \mathrm{~min}$. The reactions were visualized with 3,3'-diaminobenzidine chromogen (DAB; Dako) and counterstained with Mayer's hematoxylin. Images were acquired using the All-in-one fluorescence microscope BZ-X710 (Keyence).

Considering the important role of tumor-infiltrating lymphocytes (TILs) for the CRC prognosis, the densities of $\mathrm{CD}^{+}, \mathrm{CD}^{+}, \mathrm{CD}^{+}$, and forkhead box P3 (FOXP3) ${ }^{+} \mathrm{T}$ cells in CRC tissues were also examined. Immunohistochemistry for TILs was performed as previously described $(34,35)$. Briefly, using the Ventana Discovery XT staining system (Ventana), the sections were incubated with anti-CD3 antibody (mouse monoclonal, 518110079; Ventana), anti-CD4 (mouse monoclonal, 518108816; Ventana), anti-CD8 (mouse monoclonal, IR623; Dako; dilution 1:50), and anti-FOXP3 (mouse monoclonal, ab20034; Abcam; dilution 1:100). The microscopic images were acquired using a high-resolution digital slide scanner NanoZoomer-XR C12000 (Hamamatsu Photonics).

Immunofluorescence. Immunofluorescence was carried out on 4- $\mu \mathrm{m}$-thick FFPE sections the same way as immunohistochemistry. Sections were deparaffinized and antigen retrieval was performed in $10 \mathrm{mM}$ Tris-EDTA buffer pH 9.0 (Dako) in a microwave at $95^{\circ} \mathrm{C}$ for $20 \mathrm{~min}$. Nonspecific protein binding was blocked with Protein Block Serum-Free (Dako) for $10 \mathrm{~min}$. Sections were incubated with an antibody mixture (1:800 diluted anti-Siglec-7 antibody, and 1:400 diluted anti-CD68 antibody; mouse monoclonal, Ab783; Abcam) at $4^{\circ} \mathrm{C}$ overnight. The next day, after washing 3 times with PBS, sections were incubated with secondary antibody mixture (1:1,000 diluted anti-mouse Alexa Fluor 568 and 1:1,000 diluted anti-rabbit Alexa Fluor 488; Thermo Fisher Scientific) for $60 \mathrm{~min}$ at room temperature. Slides were counterstained with DAPI blue to visualize nuclei. All staining procedures were performed manually, and stained sections were visualized and photographed using the All-in-one fluorescence microscope BZ-X710 (KEYENCE; magnification, x200). From each section, 10 fields near the center of the tumor with the highest density of Siglec-7-positive cells and CD68-positive cells were manually selected by observers. Images were analyzed with an algorithm for positive pixel count using ImageJ software (NIH) to quantify the expression levels of Siglec-7 and CD68. The threshold intensity was set at 40 for Siglec-7 and CD68 staining. The results were presented as a percent of the total positive area to the area of the examined fields. 
Table I. Predictive markers from comprehensive proteomic analysis of tumor tissue.

\begin{tabular}{|c|c|c|c|c|c|c|c|c|}
\hline \multirow[b]{2}{*}{ Rank } & \multirow[b]{2}{*}{ Target protein } & \multicolumn{2}{|c|}{$\begin{array}{l}\text { Good prognosis } \\
\qquad(\mathrm{n}=7)\end{array}$} & \multicolumn{2}{|c|}{$\begin{array}{l}\text { Poor prognosis } \\
\qquad(\mathrm{n}=6)\end{array}$} & \multirow[b]{2}{*}{$\mid \log _{2}$ ratiol } & \multirow[b]{2}{*}{ Fisher ratio } & \multirow{2}{*}{$\begin{array}{l}\begin{array}{l}\text { Welch's } \\
\text { t-test }\end{array} \\
\text { P-value }\end{array}$} \\
\hline & & Mean & SD & Mean & SD & & & \\
\hline 1 & Sonic Hedgehog & 492.5 & 226.9 & 230.3 & 82.7 & 1.1 & 3.66 & 0.022 \\
\hline 2 & ICOSLG & 14492.5 & 14465.9 & 41296.1 & 34872.0 & 1.5 & 2.29 & 0.089 \\
\hline 3 & Lysozyme & 9204.3 & 5769.5 & 19217.0 & 12240.3 & 1.1 & 2.20 & 0.079 \\
\hline 4 & Siglec-7 & 934.8 & 511.9 & 2272.1 & 1121.8 & 1.3 & 2.00 & 0.016 \\
\hline 5 & Siglec-9 & 416.4 & 167.9 & 1158.8 & 1044.2 & 1.5 & 1.62 & 0.089 \\
\hline 6 & Fibronectin & 4794.1 & 3336.5 & 13751.4 & 8483.4 & 1.5 & 1.60 & 0.025 \\
\hline 7 & FCGR3B & 1494.4 & 827.5 & 3934.3 & 3370.6 & 1.4 & 1.42 & 0.089 \\
\hline 8 & TIMP1 & 6566.0 & 4235.2 & 14537.2 & 11608.0 & 1.1 & 1.32 & 0.117 \\
\hline 9 & LBP & 3525.2 & 2064.2 & 12051.2 & 14610.8 & 1.8 & 1.22 & 0.152 \\
\hline 10 & $\mathrm{C} 1 \mathrm{q}$ & 14432.5 & 10855.0 & 32501.8 & 22469.4 & 1.2 & 1.20 & 0.085 \\
\hline
\end{tabular}

Proteins eligible for predictive biomarkers were narrowed down by the absolute $\log _{2}$ ratio $\geq 1$ and ranked according to the Fisher ratio between the good and poor prognosis groups. Good prognosis, with overall survival of 3 years or more; poor prognosis, with overall survival of 2 years or less; SD, standard deviation; ICOSLG, inducible T cell costimulator ligand; Siglec-7, sialic acid-binding immunoglobulin-like lectin 7; Siglec-9, sialic acid-binding immunoglobulin-like lectin 9; FCGR3B, Fc fragment of IgG receptor IIIb; TIMP1, tissue inhibitor of metalloproteinase 1; LBP, lipopolysaccharide binding protein; C1q, complement component 1, q subcomponent.

Measurement of TILs. Based on the immunohistochemistry for TILs, the number of TILs was measured as previously described $(34,35)$. Briefly, intratumoral-infiltrating $\mathrm{CD}^{+}$ $\mathrm{CD}^{+}, \mathrm{CD}^{+}$and $\mathrm{FOXP}^{+}$cells were defined as TILs and their numbers were measured. Those found in the peritumoral stroma and extratumoral lymphoid structures were excluded from this analysis. A computerized image analysis system Tissue Studio (Definiens) was used to score all tumor lesions. The numbers of TILs were recorded in square millimeters as the mean number of positive cells per tumor tissue unit.

Statistical analysis. In comprehensive protein analysis, differential expression of proteins was detected using the $\log _{2}$ and Fisher ratio using Microsoft Excel 2010 (Microsoft Corporation) (36). The $\log _{2}$ ratio for a protein $k$ was calculated according to the following formula:

$$
\log _{2} \text { ratio }=\log _{2}\left(\frac{\bar{x}_{k}(\text { good prognosis group })}{\bar{x}_{k}(\text { poor prognosis group })}\right),
$$

where $\bar{x}_{k}$ is the $k^{\text {th }}$ protein of the sample mean of the good or poor prognosis group. The Fisher ratio $F$ for a protein $k$ was calculated using the following formula:

$$
\text { formula: } F(k)=\frac{\left[\bar{x}_{k}(\text { good prognosis group })-\bar{x}_{k}(\text { poor prognosis group })\right]^{2}}{\frac{1}{2}\left[s_{k}^{2}(\text { good prognosis group })+s_{k}^{2}(\text { poor prognosis group })\right]},
$$

where $s_{k}^{2}$ is the $k^{\text {th }}$ protein of the sample variance of the good or poor prognosis group.

Differences between the two groups were estimated using the Welch's t-test, which was selected for this study because recent statistical recommendations and simulation studies suggest using this test under either homoscedasticity or heteroscedasticity conditions (37). The categorical variables were compared using the $\chi^{2}$ or Fisher's exact tests. The strength of a correlation between two groups was assessed by the Spearman's rank correlation coefficient. The optimal cut-off values of the expression levels of Siglec-7, CD3, CD4, CD8, and FOXP3 were determined using either the median value or the time-dependent receiver operating characteristic (ROC) curve analysis using the Kaplan-Meier (KM) estimation method and Youden's index (sensitivity + specificity - 1) (38). The survival curves were estimated using the KM method and tested using the log-rank test. All statistical analyses were performed using $\mathrm{R}$ language for 64-bit Windows (version 3.6.1, $\mathrm{R}$ Development Core Team). $\mathrm{P}<0.05$ was considered to indicate a statistically significant difference.

\section{Results}

Selection of candidate protein to predict the efficacy of vaccination. Comprehensive analysis of the expression profiles of 1,129 proteins in 13 frozen CRC tissue samples from HLA-A*24:02-matched patients was performed. The patients were divided into good and poor prognosis groups; in 7 cases with good prognosis, the patients had OS of 3 years or more and in 6 cases with poor prognosis, the patients had OS of 2 years or less. Comparing the protein expression levels of the two groups, 23 proteins satisfied the absolute $\log _{2}$ ratio $\geq 1$ and the Fisher ratio $\geq 1$. Of the 23 proteins, Table I shows the 10 proteins with the highest Fisher ratio. The expression level of Sonic hedgehog (SHH) in the good prognosis group was significantly higher than that in the poor prognosis group $(\mathrm{P}=0.022)$. In contrast, the expression levels of Siglec-7 and fibronectin were significantly higher in the poor prognosis group than those in the good prognosis group ( $\mathrm{P}=0.016$ and 0.025 , respectively). Among them, Siglec-7 was selected as a candidate protein because of the lowest P-value. 


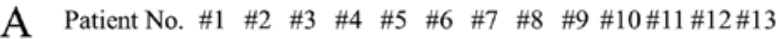
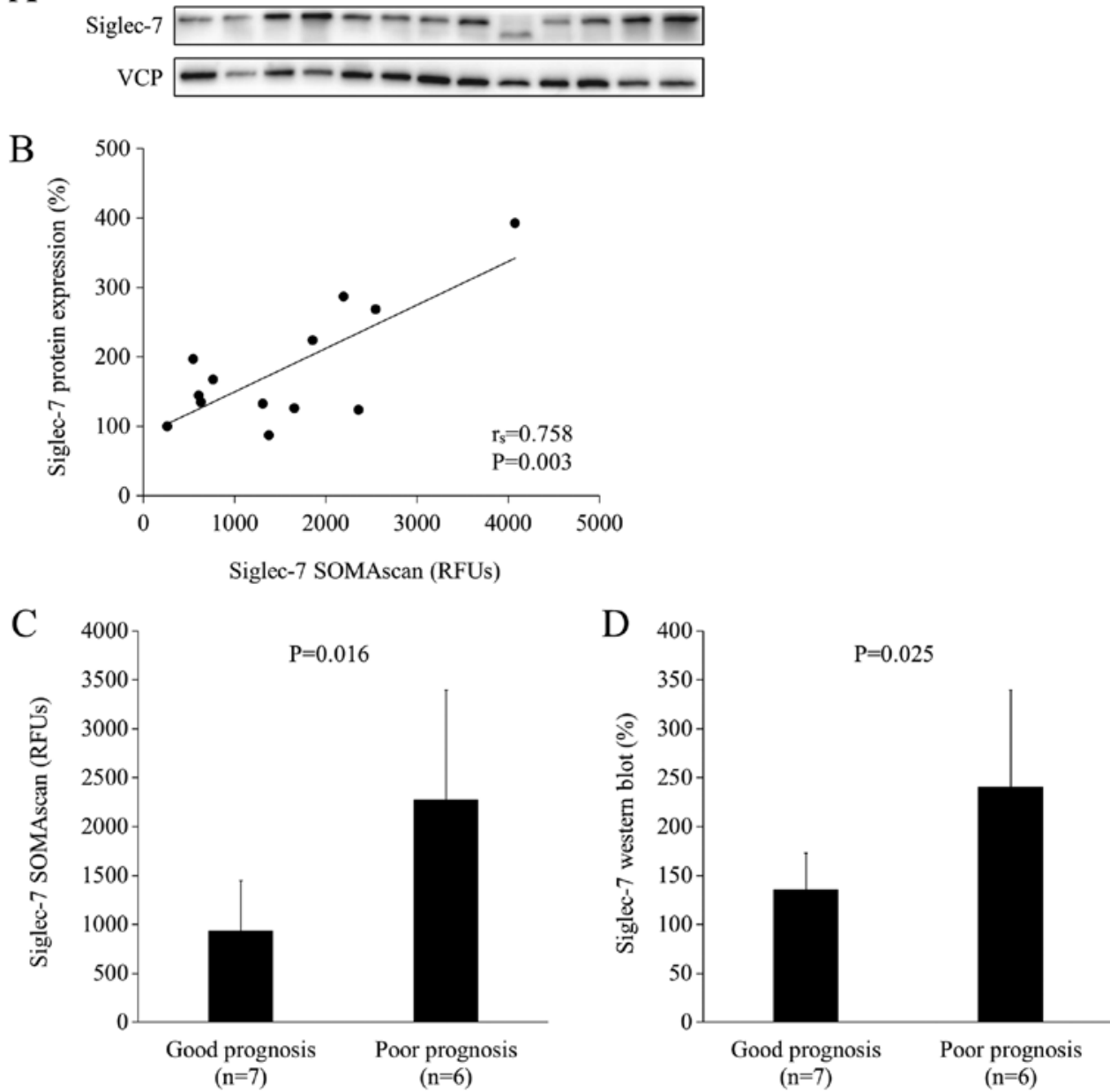

Figure 2. Confirmation of Siglec-7 expression levels and their correlation with patient prognosis. (A) The same protein samples as those in the comprehensive analysis were used in western blot analysis $(\mathrm{n}=13)$. The lower protein band in lane \#9 may reflect higher expression of the shorter isoform of Siglec-7 in the tumor tissue of patient \#9 compared with the other patients. In subsequent analysis, protein bands were quantified by densitometry and the results were presented as relative expression levels compared to the Siglec-7 expression level in patient number $1(=100 \%)$. (B) The levels of Siglec-7, as measured by SOMAscan and western blot analysis, were correlated $(n=13)$. (C) The levels of Siglec-7 measured using the SOMAscan analysis were significantly higher in the poor prognosis group. (D) The levels of Siglec-7 measured using western blot analysis were significantly higher in the poor prognosis group. Siglec-7, sialic acid-binding immunoglobulin-like lectin 7; VCP, valosin containing protein; RFU, relative fluorescence unit; OS, overall survival.

Confirmation of candidate protein expression using western blot analysis. To validate the results obtained in comprehensive analysis of SOMAscan, western blot analysis was performed using the same 13 samples as the ones used in SOMAscan analysis (Fig. 2A). As shown in Fig. 2A, the protein band in lane \#9 was lower than those in the other lanes. Siglec-7 has three isoforms, and the shorter isoform may have been highly expressed in the tumor tissue of patient \#9 compared with the other patients. The levels of Siglec-7 showed a positive correlation between SOMAscan measurements and western blot measurements ( $\mathrm{rs}=0.758, \mathrm{P}=0.00268$; Fig. 2B). The mean expression levels of Siglec-7 were significantly higher in the poor prognosis group based on both SOMAscan analysis $(\mathrm{P}=0.016)$ and in western blot analysis ( $\mathrm{P}=0.025$ ) (Fig. $2 \mathrm{C}$ and $\mathrm{D}$ ). These results indicated that the levels of Siglec-7 protein in the CRC tissue were significantly higher in patients with poor prognosis than in those with good prognosis in HLA-A*24:02-matched cohort.

Localization of Siglec-7 in tumor tissue. To identify the localization of Siglec-7 in CRC tissue, immunohistochemistry and immunofluorescence were performed. Immunohistochemistry showed that Siglec-7 was expressed in stromal cells located between or around tumor cells (Fig. 3A). Immunofluorescence showed that Siglec-7 was expressed in stromal cells which also expressed CD68 (Fig. 3B). These results indicated that Siglec-7 was expressed in intratumoral macrophages.

Validation of Siglec-7 as a predictive biomarker of vaccination. The levels of Siglec-7 expression in 63 CRC tissue samples from 32 HLA-A*24:02-matched patients and 31 HLA-A*24:02-unmatched patients were examined using immunofluorescence (Fig. 1; Table II). The levels of Siglec-7 expression ranged from 0.00001 to $7.81 \%$ (median, $0.0279 \%$ ), and from 0.0400 to $0.457 \%$ (median, $0.120 \%$ ) in HLA-A*24:02-matched and -unmatched patients, respectively. The comprehensive proteomic analysis in the present study was based on the survival of stage IV patients. Since the median OS among stage IV CRC patients is approximately 3 years, the optimal cut-off value was determined using ROC curve analysis at 36 months. This analysis was performed in HLA-A*24:02-matched patients because HLA-restricted 


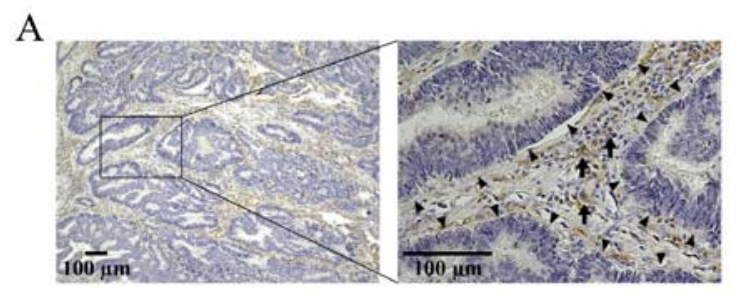

$\mathrm{B}$
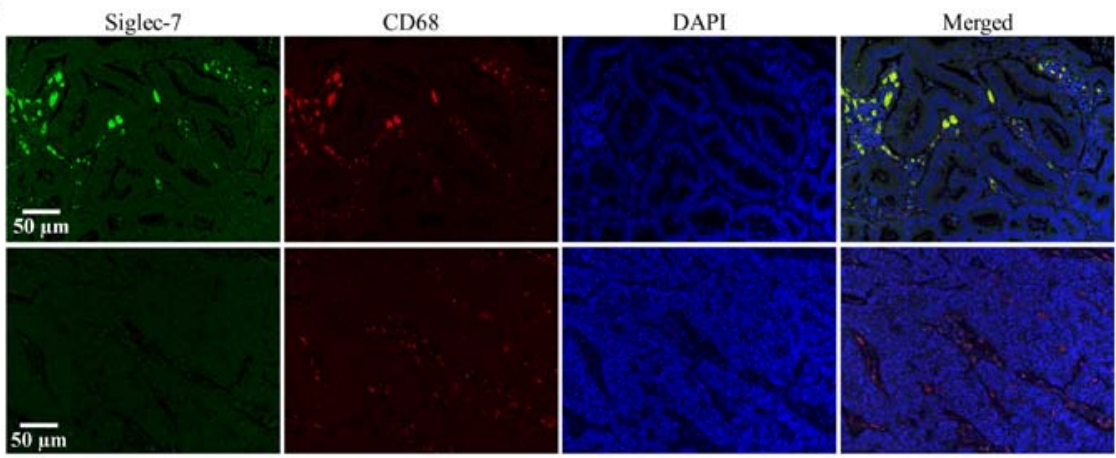

$\mathrm{C}$

HLA-A*24:02-matched

$\mathrm{D}$
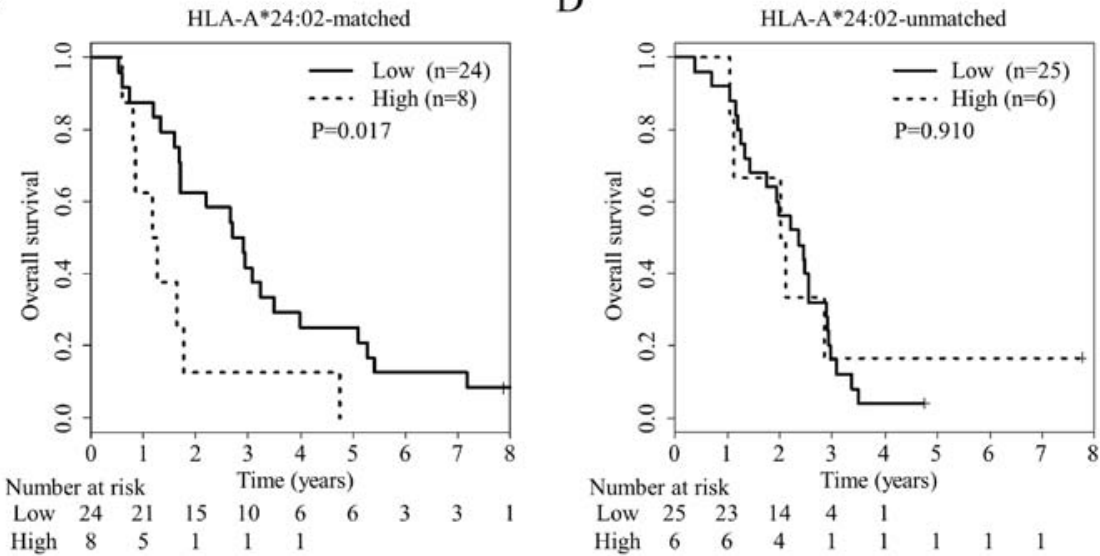

Figure 3. Siglec-7 expression in tumor microenvironment and its correlation with patient prognosis. (A) Stromal cells in the tumor area expressed Siglec-7 (indicated by arrows), whereas tumor cells did not (indicated by arrowheads). They were defined based on their locations and morphological findings. Scale bar, $100 \mu \mathrm{m}$. (B) Representative immunofluorescence images of Siglec-7 (green), CD68 (red), and nuclei (blue) in CRC tissue. Almost all of the Siglec-7 ${ }^{+}$cells expressed CD68 simultaneously, whereas there were some cells expressing only CD68. The upper row represents a tumor with high expression level of Siglec-7, and the lower one shows a tumor with low expression. Scale bar, $5 \mu \mathrm{m}$. (C) Overall survival in patients with high levels of Siglec-7 expression as detected with immunofluorescence images was significantly $(\mathrm{P}=0.017)$ shorter than that in patients with low levels of Siglec-7 expression in HLA-A*24:02-matched group. (D) There was no significant difference in overall survival between patients with high and low levels of Siglec-7 expression in HLA-A*24:02-unmatched patients. Siglec-7, sialic acid-binding immunoglobulin-like lectin 7; HLA, human leukocyte antigen; CRC, colorectal cancer; RFUs, relative fluorescence units.

peptides vaccines are theoretically effective for these patients. The cut-off value was presented as a percentage of the total positive area of Siglec-7 to the area of the examined fields. A percent of 0.213 was selected as the cut-off value for Siglec-7 expression. In the HLA-A*24:02-matched patients, the OS in patients with high Siglec-7 expression was significantly shorter than that in patients with low Siglec-7 expression ( $\mathrm{P}=0.017$; Fig. 3C). In contrast, in the HLA-A*24:02-unmatched patients, there was no significant difference in OS between patients with high or low Siglec-7 expression ( $\mathrm{P}=0.910$; Fig. 3D). In patients with low Siglec-7 expression, there was a significant difference in OS between HLA-A*24:02-matched and -unmatched patients $(\mathrm{P}=0.041 ;$ Fig. S1A), whereas there was no significant difference in patients with high Siglec-7 expression ( $\mathrm{P}=0.179$; Fig. S1B). The levels of Siglec-7 expression in tumor tissue were correlated with that of CD68 ( $r s=0.786, \mathrm{P}<0.001$; Fig. S2A). However, there was no significant difference in OS between patients with high and low levels of CD68 expression in
HLA-A*24:02-matched patients ( $\mathrm{P}=0.528$; Fig. S2B). These results indicated that Siglec-7 expression in tumor microenvironment might be a predictive biomarker of the efficacy of cancer vaccine therapy.

Relationship of TIL infiltration and prognosis with Siglec-7 expression. Because TILs have been reported as biomarkers for CRC, they were analyzed using immunohistochemistry in CRC tissue samples from 32 HLA-A*24:02-matched patients, the same as those used for Siglec-7 analysis (Fig. S3). Using ROC curve analysis at 36 months, the optimal cut-off values were determined as $440.1,133.8,52.6$ and 17.8 for $\mathrm{CD}^{+}, \mathrm{CD}^{+}$, $\mathrm{CD}^{+}$and $\mathrm{FOXP}^{+}$cell densities, respectively. There was no significant difference in OS between patients with high and low numbers of TILs including $\mathrm{CD}^{+}, \mathrm{CD}^{+}$and $\mathrm{FOXP}^{+}{ }^{+}$cells $(\mathrm{P}=0.319,0.605$ and 0.242 , respectively; Fig. 4), although there was a trend for better OS in patients with high infiltration of $\mathrm{CD}^{+}$lymphocytes ( $\mathrm{P}=0.065$; Fig. $\left.4 \mathrm{~A}\right)$. Next, the correlation 
A

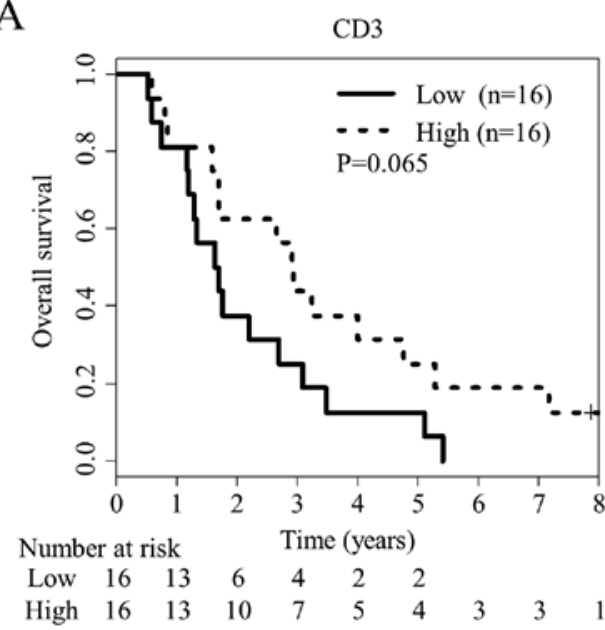

C

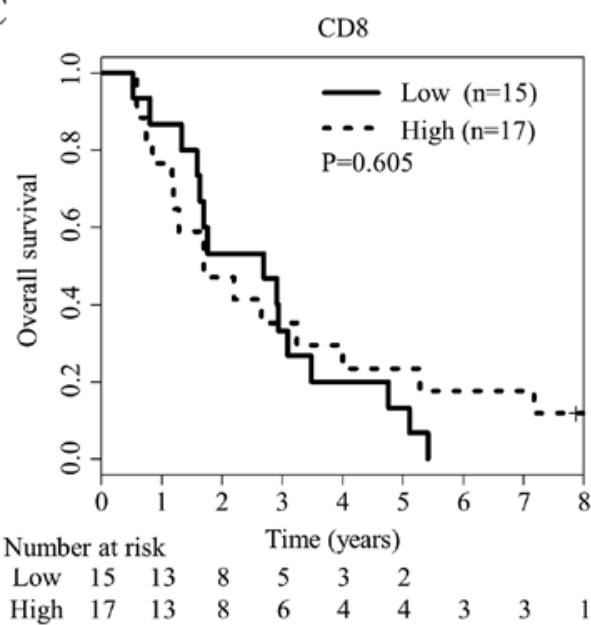

$\mathrm{B}$

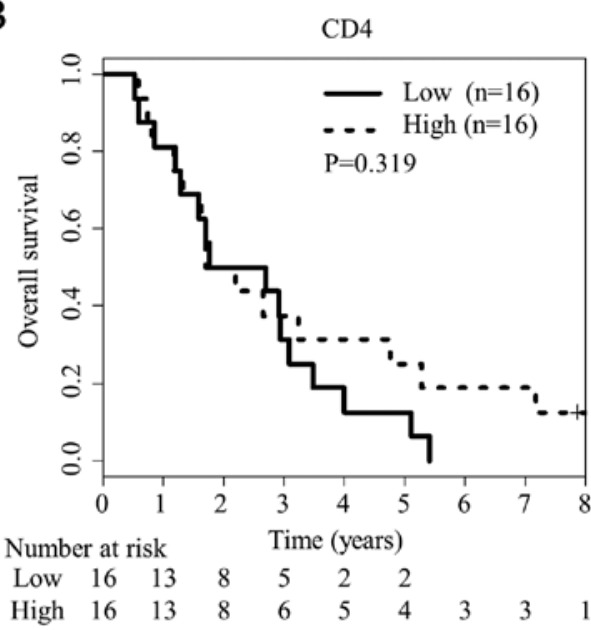

$\mathrm{D}$

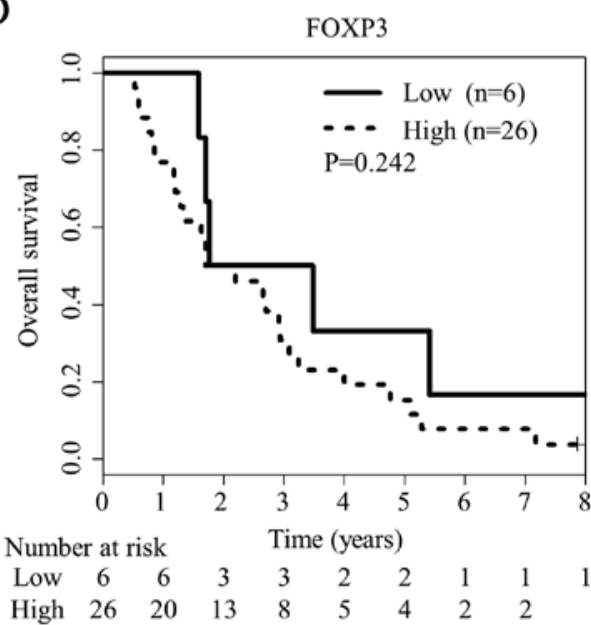

Figure 4. Overall survival according to the number of tumor-infiltrating lymphocytes (TILs). Kaplan-Meier curves for overall survival according to the number of CD3, CD4, CD8 and FOXP3 are shown. There was no significant difference in overall survival between patients with high or low numbers of TILs. (A) CD3, (B) CD4, (C) CD8, and (D) FOXP3. FOXP3, forkhead box P3.

between Siglec-7 expression and TILs in CRC tissues was examined. There were no significant associations between the levels of Siglec-7 expression detected in immunofluorescence and the numbers of $\mathrm{CD}^{+}, \mathrm{CD}^{+}, \mathrm{CD}^{+}$and $\mathrm{FOXP}^{+} \mathrm{T}$ cells in immunohistochemistry $(\mathrm{P}=0.565,0.154,0.982$ and 0.676 , respectively; Fig. 5). These findings indicated that lymphocytes and monocytes/macrophage infiltration might be independent.

\section{Discussion}

The purpose of the present study was to explore proteins as novel biomarkers to predict the efficacy of immunotherapy before treatment. First, it was demonstrated that high levels of Siglec-7 expression in tumor tissues were associated with shorter OS in patients treated with peptide vaccines for metastatic CRC. Second, it was shown that Siglec-7 was expressed in macrophages in CRC tissue. Further, there was no significant correlation between the level of Siglec-7 expression and the number of TILs in CRC tissue. These results indicated that high levels of Siglec-7 expression in intratumoral macrophages can be a negative biomarker of the vaccine treatment efficacy against metastatic CRC. To our knowledge, this is the first report showing the relationship between Siglec-7 and CRC prognosis.
In the comprehensive proteomic analysis, the good and poor prognosis groups showed significant differences in expression levels of Siglec-7, SHH, and fibronectin. SHH is a ligand for the Hedgehog signaling pathway, which is critical for embryonic development and carcinogenesis (39). Although increased expression of SHH has been associated with poor prognosis in patients with various malignancies, including CRC $(40,41)$, the present study obtained opposite results in this aspect. Furthermore, fibronectin is a ligand for many members of the integrin receptor family and it is involved in cell adhesion, migration, growth, and differentiation (42). Because the relationship between fibronectin and CRC has been already reported $(43,44)$, it was difficult to find additional roles for this protein as a biomarker in cancer vaccination against CRC. For these reasons, $\mathrm{SHH}$ and fibronectin were excluded as candidates for predictive biomarkers.

Low levels of Siglec-7 expression in tumor tissue was associated with better prognosis in HLA-A*24:02-matched patients, but not in the unmatched patients. HLA-restricted epitope peptides show theoretical antitumoral effects only in HLA-matched patients. And only HLA-A*24:02-matched patients were considered to be treated with vaccines in the present study. Therefore, the resulting difference in OS based 

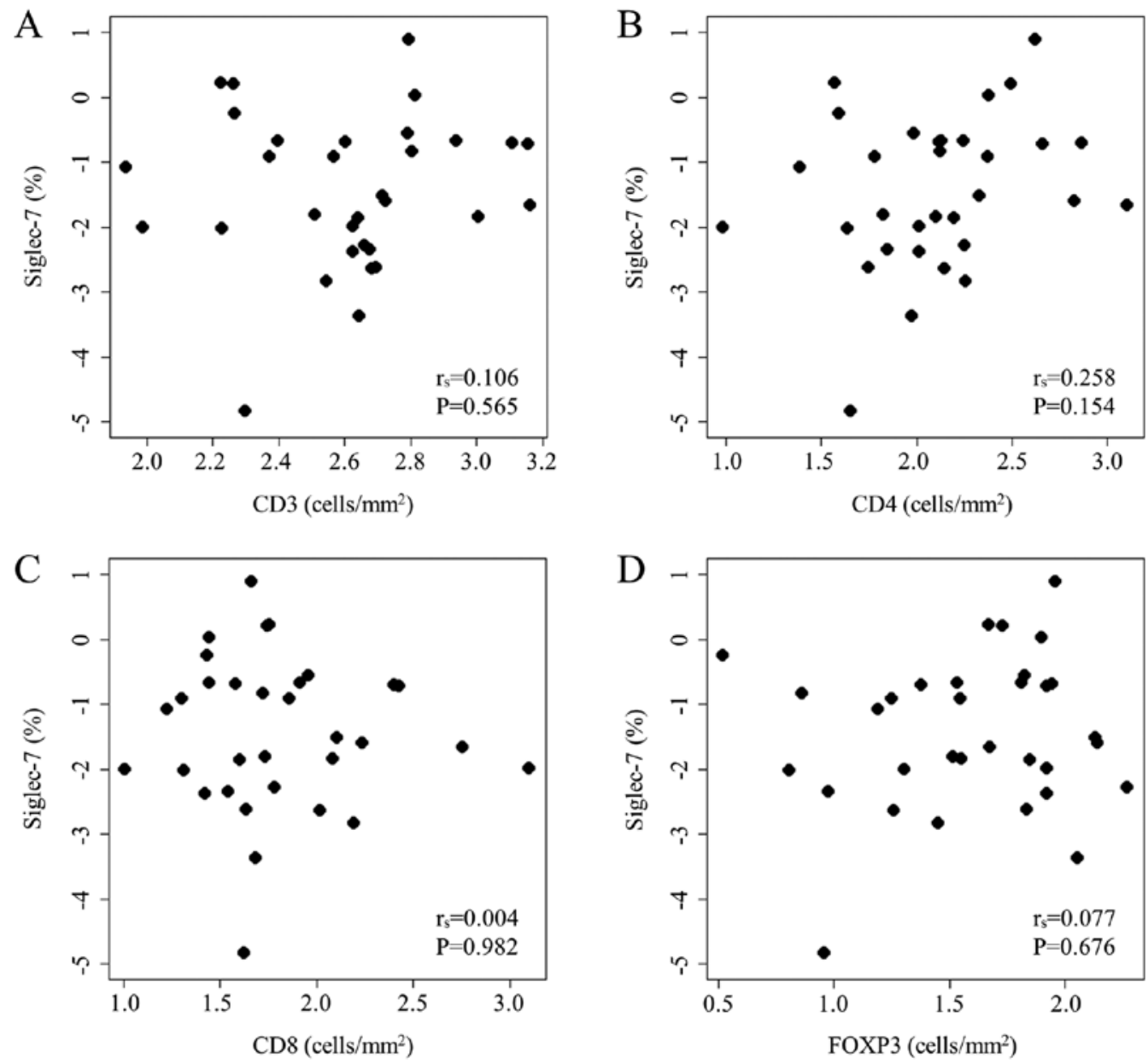

Figure 5. Relationship between Siglec-7 expression and the number of tumor-infiltrating lymphocytes (TILs). Scatterplots for CD3, CD4, CD8 and FOXP3 versus Siglec-7 are shown. The values were transformed by $\log _{10}$. There was no significant correlation between the levels of Siglec-7 expression and the numbers of TILs. (A) CD3, (B) CD4, (C) CD8, and (D) FOXP3). Siglec-7, sialic acid-binding immunoglobulin-like lectin 7; FOXP3, forkhead box P3.

on Siglec-7 expression was only in HLA-A*24:02-matched patients, indicating that Siglec-7 was not a prognostic marker for $\mathrm{CRC}$ but a predictive biomarker for cancer vaccination.

Siglec-7, a member of the CD33-related Siglecs, is mainly expressed in NK cells and monocytes/macrophages (22). The distribution of Siglec- $7^{+}$cells has been reported to differ between peripheral blood and colonic lamina propria (45). In the peripheral blood, $75 \%$ of Siglec- $7^{+}$cells were NK cells and $8 \%$ were monocytes. In colonic lamina propria, in contrast, $76 \%$ of Siglec $-7^{+}$cells were monocyte/macrophage lineages and $4 \%$ were NK cells. In this study, Siglec-7 was observed mostly in $\mathrm{CD}^{+} 8^{+}$cells in CRC tissue, thereby it was suggested that intratumoral macrophages expressed Siglec-7. The role of Siglec-7 in macrophage has been poorly explored, whereas Siglec-9, another CD33-related Siglec that shares 84\% sequence homology with Siglec-7, was reported to play an inhibitory role in macrophages (46). Specifically, Siglec-9 mediated reduction in proinflammatory cytokine tumor necrosis factor (TNF)- $\alpha$ production and potent increment in anti-inflammatory cytokine IL-10 production via ITIMs (47). Therefore, it was hypothesized that Siglec-7-expressing macrophages may mediate the reduction in secretion of proinflammatory cytokine TNF- $\alpha$ and increase in secretion of anti-inflammatory cytokine IL-10, resulting in immunosuppression of the tumor microenvironment. MSI status, another factor related to the tumor microenvironment, was also analyzed in the present study, and only one patient had MSI-high CRC (data not shown). Although the level of Siglec-7 expression was low in the MSI-high CRC, the relationship between Siglec-7 expression in CRC tissue and MSI status was not analyzed because it was statistically inappropriate.

Cancer vaccination shows antitumoral effects by introducing tumor antigen-specific cytotoxic $\mathrm{T}$ lymphocytes (CTLs). Described as the cancer-immunity cycle (48), injected HLA-restricted epitope peptides are captured and presented to $\mathrm{T}$ cells by dendritic cells via HLA molecules. Then, activated tumor antigen-specific CTLs infiltrate the tumor, recognizing and killing target cancer cells. However, CTLs may have their function inhibited by PD-L1 and immunosuppressive mediators such as IL-10 and transforming growth factor- $\beta$ in the tumor microenvironment $(49,50)$. Siglec-7 may pose an obstacle to CTLs by mediating immunosuppression of tumor microenvironment via regulation of TNF- $\alpha$ and IL-10 secretions, resulting in suppressed efficacy of vaccine treatment against metastatic CRC. These mechanisms may explain the association between high levels of Siglec-7 expression in intratumoral macrophages and poor prognosis in HLA-A*24:02-matched patients.

TILs, especially $\mathrm{CD}^{+}$and $\mathrm{CD}^{+}{ }^{+} \mathrm{T}$ cells, are prognostic biomarkers for CRC (28,51). For instance, a scoring system 
Table II. Characteristics of patients in the phase II study whose tissues were analyzed by immunofluorescence.

\begin{tabular}{|c|c|c|c|}
\hline \multirow[b]{2}{*}{ Characteristics } & \multicolumn{2}{|c|}{ HLA-A*24:02 } & \multirow[b]{2}{*}{ P-value } \\
\hline & $\begin{array}{l}\text { Matched } \\
(n=32)\end{array}$ & $\begin{array}{l}\text { Unmatched } \\
\qquad(n=31)\end{array}$ & \\
\hline \multicolumn{4}{|l|}{ Age } \\
\hline Mean & 67.9 & 64.3 & 0.069 \\
\hline Range & $47-82$ & $47-77$ & \\
\hline \multicolumn{4}{|l|}{ Sex } \\
\hline Male & 13 & 18 & 0.211 \\
\hline Female & 19 & 13 & \\
\hline \multicolumn{4}{|l|}{ Unresectable site } \\
\hline Liver & 18 & 24 & 0.300 \\
\hline Lung & 11 & 9 & \\
\hline Dissemination & 3 & 2 & \\
\hline Bone & 0 & 2 & \\
\hline Lymph node & 3 & 9 & \\
\hline Other & 3 & 1 & \\
\hline \multicolumn{4}{|c|}{$\begin{array}{l}\text { Number of metastatic } \\
\text { organs }\end{array}$} \\
\hline One & 26 & 19 & 0.068 \\
\hline Two & 6 & 8 & \\
\hline Three & 0 & 4 & \\
\hline \multicolumn{4}{|c|}{ Location of tumor } \\
\hline Colon & 22 & 24 & 0.572 \\
\hline Rectum & 10 & 7 & \\
\hline
\end{tabular}

HLA, human leukocyte antigen.

based on $\mathrm{CD}^{3+}$ and $\mathrm{CD}^{+} \mathrm{T}$ cells densities within the tumor and its invasive margin, the immunoscore, was demonstrated to be a strong prognostic factor for CRC patients $(52,53)$. In the present study, Siglec-7 expression was not associated with $\mathrm{CD}^{+}, \mathrm{CD}^{+}, \mathrm{CD}^{+}$and $\mathrm{FOXP}^{+} \mathrm{T}$ cells. It was suggested that Siglec-7 was an independent biomarker from TILs. The analysis of Siglec-7 might have led to these results by assessing macrophages rather than lymphocytes in the tumor microenvironment.

The present study, however, had several limitations. The first one is the small number of patients enrolled in this study. Second, multivariate analysis, including clinicopathological factors to adjust for confounding factors, was not performed because it was statistically inappropriate due to the small number of patients. The third limitation concerns the lack of mechanistic studies. Nonetheless, understanding the functions of Siglec-7 in the tumor environment might lead to novel immunotherapeutic strategies such as the alteration of cold tumor to hot tumor. For example, because Siglecs are endocytic receptors suitable for drug delivery, the alteration may be achieved by administering a Siglec-7-specific antibody conjugated to toxins or chemotherapeutic agents to deplete Siglec-7-expressing macrophages (54). Finally, the relationship between Siglec-7 expression and other immunologically important molecules including PD-1, PD-L1 and HLA expressions were not evaluated.

In conclusion, Siglec-7 expression in macrophages in tumor tissue might be a novel predictive biomarker for the efficacy of immunotherapy against metastatic CRC. Further studies are needed to confirm the utility of Siglec-7 as a predictive biomarker and to analyze the role of Siglec-7 in the tumor microenvironment.

\section{Acknowledgements}

The authors would like to thank Ms. Hiroko Takenouchi (Department of Translational Research and Developmental Therapeutics against Cancer, Yamaguchi University School of Medicine) for her technical support.

\section{Funding}

This study was performed as a research program of the Project for Development of Innovative Research on Cancer Therapeutics (P-DIRECT; grant no. 11039020) and The Japan Agency for Medical Research and Development (AMED; grant no. 15cm0106085h0005). This study was supported in part by a grant for Leading Advanced Projects for Medical Innovation (LEAP; grant no. 16am0001006h0003) from the Japan Agency for Medical Research and Development.

\section{Availability of data and materials}

The datasets used and/or analyzed during the current study are available from the corresponding author on reasonable request.

\section{Authors' contributions}

KY, SH and HN designed the study. KY, NS, MX, NF, RT, SY, STo, SM, HM, YT, SK, YS, YW, MI, STa, TI, TU, YHo, HK, TF and YK contributed to patient recruitment and collection of data, and analysis and interpretation of data. KY, YN, HO and YHa performed the statistical analysis. KY, SH and $\mathrm{HN}$ wrote the manuscript. All authors read and approved the final manuscript.

\section{Ethics approval and consent to participate}

The study was carried out according to the Declaration of Helsinki on experimentation on human subjects and was approved by the Institutional Ethics Review Boards of Yamaguchi University (approval number: H20-102; Clinical Trials Registry: UMIN000001791). Written informed consent for participation in this study was obtained from each patient.

\section{Patient consent for publication}

Written informed consent for publication was obtained from each patient at the time of enrollment.

\section{Competing interests}

The authors declare that they have no competing interests. 


\section{References}

1. Siegel RL, Miller KD and Jemal A: Cancer statistics, 2020. CA Cancer J Clin 70: 7-30, 2020.

2. Loupakis F, Cremolini C, Masi G, Lonardi S, Zagonel V. Salvatore L, Cortesi E, Tomasello G, Ronzoni M, Spadi R, et al: Initial therapy with FOLFOXIRI and bevacizumab for metastatic colorectal cancer. N Engl J Med 371: 1609-1618, 2014.

3. Sanchez-CastañónM,ErTK,BujandaLandHerreros-VillanuevaM: Immunotherapy in colorectal cancer: What have we learned so far? Clin Chim Acta 460: 78-87, 2016

4. Myint ZW and Goel G: Role of modern immunotherapy in gastrointestinal malignancies: A review of current clinical progress. J Hematol Oncol 10: 86, 2017.

5. Hazama S, Tamada K, Yamaguchi Y, Kawakami Y and Nagano H: Current status of immunotherapy against gastrointestinal cancers and its biomarkers: Perspective for precision immunotherapy. Ann Gastroenterol Surg 2: 289-303, 2018.

6 . Kono K: Advances in cancer immunotherapy for gastroenterological malignancy. Ann Gastroenterol Surg 2: 244-245, 2018.

7. Le DT, Durham JN, Smith KN, Wang H, Bartlett BR, Aulakh LK Lu S, Kemberling H, Wilt C, Luber BS, et al: Mismatch repair deficiency predicts response of solid tumors to PD-1 blockade. Science 357: 409-413, 2017

8. Overman MJ, McDermott R, Leach JL, Lonardi S, Lenz HJ, Morse MA, Desai J,Hill A, Axelson M, Moss RA, et al: Nivolumab in patients with metastatic DNA mismatch repair-deficient or microsatellite instability-high colorectal cancer (CheckMate 142): An open-label, multicentre, phase 2 study. Lancet Oncol 18 : 1182-1191, 2017

9. Nagorsen D and Thiel E: Clinical and immunologic responses to active specific cancer vaccines in human colorectal cancer. Clin Cancer Res 12: 3064-3069, 2006.

10. Le DT, Uram JN, Wang H, Bartlett BR, Kemberling H, Eyring AD, Skora AD, Luber BS, Azad NS, Laheru D, et al: PD-1 blockade in tumors with mismatch-repair deficiency. N Engl J Med 372: 2509-2520, 2015

11. Duan Q, Zhang H, Zheng J and Zhang L: Turning cold into hot: Firing up the tumor microenvironment. Trends Cancer 6: 605-618, 2020

12. Hazama S, Nakamura Y, Takenouchi H, Suzuki N, Tsunedomi R, Inoue $\mathrm{Y}$, Tokuhisa Y, Iizuka $\mathrm{N}$, Yoshino $\mathrm{S}$, Takeda $\mathrm{K}$, et al: A phase I study of combination vaccine treatment of five therapeutic epitope-peptides for metastatic colorectal cancer; safety, immunological response, and clinical outcome. J Transl Med 12: 63, 2014.

13. Hazama S, Nakamura Y, Tanaka H, Hirakawa K, Tahara K, Shimizu R, Ozasa H, Etoh R, Sugiura F, Okuno K, et al: A phase II study of five peptides combination with oxaliplatin-based chemotherapy as a first-line therapy for advanced colorectal cancer (FXV study). J Transl Med 12: 108, 2014

14. Hazama S, Takenouchi H, Tsunedomi R, Iida M, Suzuki N, Iizuka N, Inoue Y, Sakamoto K, Nakao M, Shindo Y, et al: Predictive biomarkers for the outcome of vaccination of five therapeutic epitope peptides for colorectal cancer. Anticancer Res 34: 4201-4205, 2014

15. Shindo Y, Hazama S, Nakamura Y, Inoue Y, Kanekiyo S, Suzuki N, Takenouchi H, Tsunedomi R, Nakajima M, Ueno T, et al: miR-196b, miR-378a and miR-486 are predictive biomarkers for the efficacy of vaccine treatment in colorectal cancer. Oncol Lett 14: 1355-1362, 2017.

16. Kijima T, Hazama S, Tsunedomi R, Tanaka H, Takenouchi $\mathrm{H}$, Kanekiyo S, Inoue Y, Nakashima M, Iida M, Sakamoto K, et al: MicroRNA-6826 and -6875 in plasma are valuable non invasive biomarkers that predict the efficacy of vaccine treatment agains metastatic colorectal cancer. Oncol Rep 37: 23-30, 2017.

17. Tanaka H, Hazama S, Iida M, Tsunedomi R, Takenouchi H, Nakajima M, Tokumitsu Y, Kanekiyo S, Shindo Y, Tomochika S, et al: miR-125b-1 and miR-378a are predictive biomarkers for the efficacy of vaccine treatment against colorectal cancer. Cancer Sci 108: 2229-2238, 2017.

18. Kitahara M, Hazama S, Tsunedomi R, Takenouchi H, Kanekiyo S, Inoue Y, Nakajima M, Tomochika S, Tokuhisa Y, Iida M, et al: Prediction of the efficacy of immunotherapy by measuring the integrity of cell-free DNA in plasma in colorectal cancer. Cancer Sci 107: 1825-1829, 2016

19. Crocker PR, Paulson JC and Varki A: Siglecs and their roles in the immune system. Nat Rev Immunol 7: 255-266, 2007.

20. Fraschilla I and Pillai S: Viewing Siglecs through the lens of tumor immunology. Immunol Rev 276: 178-191, 2017.
21. Matsumoto T, Takahashi N, Kojima T, Yoshioka Y, Ishikawa J, Furukawa K, Ono K, Sawada M, Ishiguro N and Yamamoto A: Soluble Siglec-9 suppresses arthritis in a collagen-induced arthritis mouse model and inhibits M1 activation of RAW264.7 macrophages. Arthritis Res Ther 18: 133, 2016.

22. Nicoll G, Ni J, Liu D, Klenerman P, Munday J, Dubock S, Mattei MG and Crocker PR: Identification and characterization of a novel siglec, siglec-7, expressed by human natural killer cells and monocytes. J Biol Chem 274: 34089-34095, 1999.

23. Daly J, Carlsten M and O'Dwyer M: Sugar free: Novel immunotherapeutic approaches targeting siglecs and sialic acids to enhance natural killer cell cytotoxicity against cancer. Front Immunol 10: 1047, 2019.

24. Suda T, Tsunoda T, Daigo Y, Nakamura Y and Tahara H: Identification of human leukocyte antigen-A24-restricted epitope peptides derived from gene products upregulated in lung and esophageal cancers as novel targets for immunotherapy. Cancer Sci 98: 1803-1808, 2007.

25. Shimokawa T, Matsushima S, Tsunoda T, Tahara H, Nakamura Y and Furukawa Y: Identification of TOMM34, which shows elevated expression in the majority of human colon cancers, as a novel drug target. Int J Oncol 29: 381-386, 2006.

26. Uchida N, Tsunoda T, Wada S, Furukawa Y, Nakamura Y and Tahara H: Ring finger protein 43 as a new target for cancer immunotherapy. Clin Cancer Res 10: 8577-8586, 2004.

27. Ishizaki H, Tsunoda T, Wada S, Yamauchi M, Shibuya M and Tahara $\mathrm{H}$ : Inhibition of tumor growth with antiangiogenic cancer vaccine using epitope peptides derived from human vascular endothelial growth factor receptor 1. Clin Cancer Res 12: 5841-5849, 2006.

28. Wada S, Tsunoda T, Baba T, Primus FJ, Kuwano H, Shibuya M and Tahara $\mathrm{H}$ : Rationale for antiangiogenic cancer therapy with vaccination using epitope peptides derived from human vascular endothelial growth factor receptor 2. Cancer Res 65: 4939-4946, 2005.

29. Nakashima-Nakasuga C, Hazama S, Suzuki N, Nakagami Y, Xu M, Yoshida S, Tomochika S, Fujiwara N, Matsukuma S, Matsui H, et al: Serum LOX-1 is a novel prognostic biomarker of colorectal cancer. Int J Clin Oncol 25: 1308-1317, 2020.

30. Fujiwara N, Usui T, Ohama T and Sato K: Regulation of beclin 1 protein phosphorylation and autophagy by protein phosphatase 2A (PP2A) and death-associated protein kinase 3 (DAPK3). J Biol Chem 291: 10858-10866, 2016.

31. Schneider CA, Rasband WS and Eliceiri KW: NIH Image to ImageJ: 25 years of image analysis. Nat Methods 9: 671-675, 2012.

32. Enjoji S, Yabe R, Tsuji S, Yoshimura K, Kawasaki H, Sakurai M, Sakai Y, Takenouchi $\mathrm{H}$, Yoshino S, Hazama S, et al: Stemness is enhanced in gastric cancer by a SET/PP2A/E2F1 axis. Mol Cancer Res 16: 554-563, 2018.

33. Yabe R, Tsuji S, Mochida S, Ikehara T, Usui T, Ohama T and Sato K: A stable association with PME-1 may be dispensable for PP2A demethylation - implications for the detection of PP2A methylation and immunoprecipitation. FEBS Open Bio 8: 1486-1496, 2018

34. Galon J, Costes A, Sanchez-Cabo F, Kirilovsky A, Mlecnik B, Lagorce-Pagès C, Tosolini M, Camus M, Berger A, Wind P, et al: Type, density, and location of immune cells within human colorectal tumors predict clinical outcome. Science 313: 1960-1964, 2006

35. Kuwahara T, Hazama S, Suzuki N, Yoshida S, Tomochika S, Nakagami Y, Matsui H, Shindo Y, Kanekiyo S, Tokumitsu Y, et al: Intratumoural-infiltrating $\mathrm{CD}^{+}{ }^{+}$and $\mathrm{FOXP}^{+}{ }^{+} \mathrm{T}$ cells as strong positive predictive markers for the prognosis of resectable colorectal cancer. Br J Cancer 121: 659-665, 2019.

36. Iizuka N, Oka M, Yamada-Okabe H, Nishida M, Maeda Y, Mori N, Takao T, Tamesa T, Tangoku A, Tabuchi $\mathrm{H}$, et al: Oligonucleotide microarray for prediction of early intrahepatic recurrence of hepatocellular carcinoma after curative resection. Lancet 361: 923-929, 2003.

37. Zimmerman DW: Some properties of preliminary tests of equality of variances in the two-sample location problem. J Gen Psychol 123: 217-231, 1996.

38. Kamarudin AN, Cox T and Kolamunnage-Dona R: Time-dependent ROC curve analysis in medical research: Current methods and applications. BMC Med Res Methodol 17: 53, 2017.

39. Yoshikawa K, Shimada M, Miyamoto H, Higashijima J, Miyatani T, Nishioka M, Kurita N, Iwata T and Uehara H: Sonic hedgehog relates to colorectal carcinogenesis. J Gastroenterol 44: $1113-1117,2009$ 
40. Xu M, Li X, Liu T, Leng A and Zhang G: Prognostic value of hedgehog signaling pathway in patients with colon cancer. Med Oncol 29: 1010-1016, 2012.

41. Maréchal R, Bachet JB, Calomme A, Demetter P, Delpero JR, Svrcek M, Cros J, Bardier-Dupas A, Puleo F, Monges G, et al: Sonic hedgehog and Gli1 expression predict outcome in resected pancreatic adenocarcinoma. Clin Cancer Res 21: 1215-1224, 2015.

42. Pankov R and Yamada KM: Fibronectin at a glance. J Cell Sci 115: 3861-3863, 2002

43. Yi W, Xiao E, Ding R, Luo P and Yang Y: High expression of fibronectin is associated with poor prognosis, cell proliferation and malignancy via the $\mathrm{NF}-\mathrm{\kappa B} / \mathrm{p} 53$-apoptosis signaling pathway in colorectal cancer. Oncol Rep 36: 3145-3153, 2016.

44. Inufusa H, Nakamura M, Adachi T, Nakatani Y, Shindo K, Yasutomi $\mathrm{M}$ and Matsuura $\mathrm{H}$ : Localization of oncofetal and normal fibronectin in colorectal cancer. Correlation with histologic grade, liver metastasis, and prognosis. Cancer 75 2802-2808, 1995.

45. Miyazaki K, Sakuma K, Kawamura YI, Izawa M, Ohmori K, Mitsuki M, Yamaji T, Hashimoto Y, Suzuki A, Saito Y, et al: Colonic epithelial cells express specific ligands for mucosal macrophage immunosuppressive receptors siglec-7 and -9 . J Immunol 188: 4690-4700, 2012.

46. Dharmadhikari G, Stolz K, Hauke M, Morgan NG, Varki A de Koning E, Kelm S and Maedler K: Siglec-7 restores $\beta$-cell function and survival and reduces inflammation in pancreatic islets from patients with diabetes. Sci Rep 7: 45319, 2017.

47. Ando M, Tu W, Nishijima K and Iijima S: Siglec-9 enhances IL-10 production in macrophages via tyrosine-based motifs. Biochem Biophys Res Commun 369: 878-883, 2008.
48. Chen DS and Mellman I: Oncology meets immunology: The cancer-immunity cycle. Immunity 39: 1-10, 2013.

49. Dong H, Strome SE, Salomao DR, Tamura H, Hirano F, Flies DB, Roche PC, Lu J, Zhu G, Tamada K, et al: Tumor-associated B7-H1 promotes T-cell apoptosis: A potential mechanism of immune evasion. Nat Med 8: 793-800, 2002.

50. Vermaelen K: Vaccine strategies to improve anti-cancer cellular immune responses. Front Immunol 10: 8, 2019.

51. Mlecnik B, Tosolini M, Kirilovsky A, Berger A, Bindea G, Meatchi T, Bruneval P, Trajanoski Z, Fridman WH, Pagès F, et al: Histopathologic-based prognostic factors of colorectal cancers are associated with the state of the local immune reaction. J Clin Oncol 29: 610-618, 2011.

52. Galon J, Mlecnik B, Bindea G, Angell HK, Berger A, Lagorce C, Lugli A, Zlobec I, Hartmann A, Bifulco C, et al: Towards the introduction of the 'Immunoscore' in the classification of malignant tumours. J Pathol 232: 199-209, 2014.

53. Pagès F, Mlecnik B, Marliot $F$, Bindea G, Ou FS, Bifulco C, Lugli A, Zlobec I, Rau TT, Berger MD, et al: International validation of the consensus Immunoscore for the classification of colon cancer: A prognostic and accuracy study. Lancet 391: 2128-2139, 2018.

54. O'Reilly MK and Paulson JC: Siglecs as targets for therapy in immune-cell-mediated disease. Trends Pharmacol Sci 30: 240-248, 2009

(i) (9) This work is licensed under a Creative Commons C) Attribution-NonCommercial-NoDerivatives 4.0 International (CC BY-NC-ND 4.0) License. 